\title{
Editorial Overview
}

\author{
Jeffrey Skolnick and \\ Center for the Study of Systems Biology, Georgia, Institute of Technology, Atlanta, GA 30318, e- \\ mail: skolnick@gatech.edu \\ Richard A. Friesner \\ Department of Chemistry, Columbia University, New York, 10027, USA, email: \\ rich@chem.columbia.edu
}

\begin{abstract}
The application of computational methods to the modeling of protein structure, dynamics, and function has made rapid progress over the past decade. The combination of the exponential reduction in the cost/performance of computational hardware with continually improving algorithms, energy functions, and software implementations has yielded significant improvements in the ability to achieve better accuracy and reliability in structural models of ever more complex systems and to address their dynamics at much longer timescales. These advances in turn have yielded insights that are increasingly relevant with regard to biological mechanisms at an atomic scale. Indeed, as illustrated in several of the articles below, in a number of key areas, computation has reached the point where it has become a full partner with experiment in understanding function; the work on ion channels, discussed by Schulten and coworkers below, is a particularly striking example.
\end{abstract}

The articles in this volume cover a range of methodologies, starting from atomistic, explicit solvent molecular dynamics simulations, and continuing through various intermediate levels of rigorous to approximate models based on statistical potentials and residue levels of resolution, aimed at understanding fold and function on a relatively coarse grained scale. The most fine-grained approaches generally have the highest computational cost, but also the highest level of structural and energetic resolution. The more approximate models and approaches sacrifice some detail in return for the ability to obtain results more quickly, ultimately yielding tools applicable on a genomic scale with current computational resources. Some approaches initially model the system of interest on a coarse-grained level and then add the details (e.g. as in the prediction of protein structure as described by Yang below) once the relevant region of conformational space is found by the coarse-grained approach.

The first two articles, from the Shaw and Schulten laboratories, focus on long time molecular dynamics simulations. The Shaw article is roughly evenly divided between a discussion of methodology and descriptions of illustrative applications, including protein folding, simulation of protein conformational dynamics (e.g. in kinases), and diffusion of small molecules through membrane channels. A particularly exciting prospect is the development of special purpose hardware and software enabling millisecond simulations to be achieved, which would open up a much wider range of biologically relevant events to atomistic simulation technology. The Schulten article is primarily focused on simulation results for membrane channels, including both ion channels and aquaporins, but also discusses the impact of advancing technology on the ability to tackle these challenging problems. A number of interesting examples are

\footnotetext{
Publisher's Disclaimer: This is a PDF file of an unedited manuscript that has been accepted for publication. As a service to our customers we are providing this early version of the manuscript. The manuscript will undergo copyediting, typesetting, and review of the resulting proof before it is published in its final citable form. Please note that during the production process errors may be discovered which could affect the content, and all legal disclaimers that apply to the journal pertain.
} 
considered in some detail, and comparisons of theory with experiment (and how theory and experiment can be used together to understand mechanism) are emphasized. The impact of improved simulation models, including explicit treatment of polarization in the molecular mechanics force field, is briefly discussed.

Complementary to the Schulten article is the more methodological contribution from the Voth laboratory that describes both a general, rigorous "bottom up" approach for developing coarsegrained membrane and protein models from detailed atomic models, as well as more phenomenological "top down" approaches to coarse-graining. Particularly noteworthy in this regard is the MARTINI method of Sansom et al that employs a knowledge-based parameterization. Moving to even more mesoscopic models, there are elastic network membrane models. The focus is on the way that atomic interactions are incorporated into multiscale simulation approaches to access larger systems and longer time scales than can be achieved by brute force simulations at atomic detail. These are general methodologies that will likely be exploited in a variety of contexts.

Very similar ideas permeate the article from the Yang laboratory that describes the status of the field of protein structure prediction as assessed by CASP7 and CASP8 (community wide blind tests of protein structure prediction methods). He describes the range of utility of various quality protein structures for protein functional inference, ligand screening and molecular replacement in X-ray crystallography. The most successful protein methods are hierarchicalviz. they assemble an approximate predicted protein structure in a coarse-grained protein representation and then add the atomic details. This is a particularly powerful approach, as it is likely that the library of single domain templates is complete (e.g. there were no new folds in CASP8); the unsolved problem is to identify such structural templates when the protein of interest is, at best, evolutionarily quite distant from the template. While progress at the coarsegrained level has been made in the ability to refine protein structures over their best starting template structures, success in detailed atomic model refinement has been more modest as there are issues with physics-based energy functions. Over the short term, progress is likely to be made by combining physics-based with knowledge-based potentials.

Articles from the Jacobson and Vajda laboratories highlight the use of computational techniques to address key post-translational interactions and modifications of protein structures that are vital to cell function. Jacobson discusses several ubiquitous forms of chemical modification, with the greatest focus on phosphorylation, a dominant mechanism for activating signaling cascades (e.g. via kinases). The goal is to understand the structural changes induced by the addition of the phosphate group, for example alterations in loop conformations, and to be able to predict these changes using computational techniques. A number of approaches are discussed, beginning with explicit solvent molecular dynamics simulations, but also including conformational search methods employing continuum solvation models, which are able to explore larger regions of phase space in considerably less computation time. The ability of such methods to accurately predict loop and side chain conformations, even for relatively long loops, has been demonstrated in previous work. Vajda considers protein-protein docking, essential for assembling the complex molecular machines that perform many vital cell functions. Vajda considers protein-protein docking, essential for assembling the complex molecular machines that perform many vital cell functions. He summarizes recent results from CAPRI (a blind test comparing docking calculations to experimental data), provides an overview of various models employed for this purpose (which include continuum solvation treatments but also knowledge-based potentials with, in some cases, coarser resolution), and discusses algorithms used to generate the structural predictions. Once again, composite methods, starting with lower resolution and working up to strategies involving explicit sampling of side chains, are likely to be necessary for ultimate solution of this very difficult problem; meanwhile, as summarized in the article, significant progress has been made. 
Turning to the biologically very important problem of DNA-protein recognition, the Honig Laboratory continues the theme of employing detailed atomic/coarse grained modeling. In particular, they focus on the intrinsic structure of DNA. They describe DNA sequence dependent structural variations that can play an essential role in how a protein recognizes DNA. To capture these very subtle effects, a detailed atomic description is required. These insights have important implications as to how a protein recognizes its specific binding site in, what to a first approximation, looks like a uniform DNA structure. On the other hand, coarse-grained simulations have begun to describe the function of larger assemblies such as nucleosome positioning. Just as for protein structure refinement, improved detailed atomic potentials that are carefully benchmarked on DNA and DNA/protein systems are need. Again, they argue that progress in understanding the mechanism of DNA-protein recognition will result from a multiscale combination of experimental, knowledge-based and physics-based approaches.

This issue of COSB focuses on a wide variety of systems ranging from individual proteins and associated posttranslational modifications to protein-protein interactions to DNA-protein interactions to protein-membrane systems that all share many common features. To better understand their behavior, multiscale approaches are required. This partly reflects intrinsic computational limitations as well as the possibility that knowledge-based approaches appear to better capture coarse-grained features, while contemporary physics-based approaches can capture the subtle details. There is the need to develop better sampling schemes as well as special purpose hardware/software that will enable larger systems to be fully sampled at longer times. There remain significant issues with the underlying knowledge-based and physics-based force fields. Nevertheless, despite these limitations, significant progress has been made in modeling macromolecules and their assemblies. This paves the way to think about modeling subcellular compartments to understand cellular collective behavior. While much remains to be done before modeling of cells becomes a reality, we are witnessing the early development of approaches that hold the promise of making significant progress towards achieving this goal.

While continued progress in multiscale modeling will be essential to tackling a wide range of biologically relevant problems, it is also the case that much work remains to be done in traditional, atomistic modeling at the level of highest accuracy. Some critical technologies, such as structure based drug design, will not become true enabling technology platforms until high resolution structural prediction, and accurate calculation of energy differences (e.g. protein-ligand binding affinities), become more reliable and precise. Fundamental problems in force field development, continuum solvation modeling, and sampling effectiveness remain as key issues in this area. The articles in this volume report encouraging progress in this area, but further effort is needed to reach the goals required for robust practical application.

\section{Biographies}

Jeffrey Skolnick's lab works on computational biology, systems biology, and cancer metabolomics. He has developed algorithms for the prediction of protein structure and function from protein sequence, the automatic assignment of enzymes to metabolic pathways, the prediction of protein-protein interactions and quaternary structure, the prediction of membrane protein tertiary structure, the prediction of DNA-protein interactions, and the identification of small molecule ligands for drug discovery. He then applies these approaches to entire proteomes, e.g. in the prediction of the tertiary structures of all human GPCRs. He is also interested in understanding the physical factors responsible for the universe of protein structures

Richard Friesner works in a number of different areas of theoretical and computational chemistry, including methodology and software development, materials science, and modeling of biological systems. His work in protein modeling is focused on high resolution structural 
prediction and refinement, including loop prediction, side chain prediction, and protein-ligand docking and prediction of binding affinities. The methods used employ both continuum and explicit solvation models, and incorporate conformational searching and molecular dynamics techniques. 